Article

\title{
Nanocomposite Materials with Poly(L-lactic Acid) and Transition-Metal Dichalcogenide Nanosheets 2D-TMDCs $\mathrm{WS}_{2}$
}

\author{
Mohammed Naffakh ${ }^{1, * \mathbb{D}}$, Miriam Fernández ${ }^{1,2}$, Peter S. Shuttleworth $^{2} \mathbb{D}$, Ana M. García ${ }^{1}$ (D) \\ and Diego A. Moreno 1,3 \\ 1 Escuela Técnica Superior de Ingenieros Industriales, Universidad Politécnica de Madrid (ETSII-UPM), \\ José Gutiérrez Abascal 2, 28006 Madrid, Spain; miriam.fernandezgall@alumnos.upm.es (M.F.); \\ ana.garcia.ruiz@upm.es (A.M.G.); diego.moreno@upm.es (D.A.M.) \\ 2 Instituto de Ciencia y Tecnología de Polímeros (ICTP-CSIC), Juan de la Cierva 3, 28006 Madrid, Spain; \\ peter@ictp.csic.es \\ 3 Facultad de Farmacia, Universidad de Castilla-La Mancha (FF-UCLM), Avda. Dr. José María Sánchez Ibañez \\ s/n, E-02071 Albacete, Spain \\ * Correspondence: mohammed.naffakh@upm.es; Tel.: +34-913-363-164 \\ Academic Editor: Tzong-Ming Wu \\ Received: 29 October 2020; Accepted: 13 November 2020; Published: 16 November 2020

\begin{abstract}
Layered transition-metal dichalcogenides (TMDCs) based on tungsten disulfide nanosheets $\left(2 \mathrm{D}-\mathrm{WS}_{2}\right)$ were introduced via melt processing into poly(L-lactic acid) (PLLA) to generate PLLA/2D-WS ${ }_{2}$ nanocomposite materials. The effects of the $2 \mathrm{D}-\mathrm{WS}_{2}$ on the morphology, crystallization, and biodegradation behavior of PLLA were investigated. In particular, the non-isothermal melt-crystallization of neat PLLA and PLLA/2D-WS 2 nanocomposites were analyzed in detail by varying both the cooling rate and $2 \mathrm{D}-\mathrm{WS}_{2}$ loading. The kinetic parameters of PLLA chain crystallization are successfully described using the Liu model. It was found that the PLLA crystallization rate was reduced with $2 \mathrm{D}-\mathrm{WS}_{2}$ incorporation, while the crystallization mechanism and crystal structure of PLLA remained unchanged in spite of nanoparticle loading. This was due to the PLLA chains not being able to easily adsorb on the $\mathrm{WS}_{2}$ nanosheets, hindering crystal growth. In addition, from surface morphology analysis, it was observed that the addition of $2 \mathrm{D}-\mathrm{WS}_{2}$ facilitated the enzymatic degradation of poorly biodegradable PLLA using a promising strain of actinobacteria, Lentzea waywayandensis. The identification of more suitable enzymes to break down PLLA nanocomposites will open up new avenues of investigation and development, and it will also lead to more environmentally friendly, safer, and economic routes for bioplastic waste management.
\end{abstract}

Keywords: 2D-WS 2 ; PLLA; nanomaterials; morphology; crystallization kinetics; biodegradation

\section{Introduction}

Poly(L-lactic acid) (PLLA) is a highly versatile, biodegradable, aliphatic polyester derived from $100 \%$ renewable resources, such as corn and sugar beets. This bioplastic offers great promise in a wide range of environmental and biomedical applications due to its favorable biodegradability, renewability, reasonably good mechanical properties, and versatile fabrication methods [1-3]. PLLA and its degradation products, namely $\mathrm{H}_{2} \mathrm{O}$ and $\mathrm{CO}_{2}$, are neither toxic nor carcinogenic to the human body, making it an excellent material for biomedical applications including sutures, clips, and drug delivery systems (DDS). Furthermore, PLLA can be processed by film casting, extrusion, blow molding, and fiber spinning due to its better thermal processability in comparison to other biomaterials such as poly(ethylene glycol) (PEG), poly(hydroxyalkanoates) (PHAs), and poly(e-caprolactone) (PCL) [4]. 
In spite of its excellent balance of properties, its commercial viability has historically been limited by high production costs and poor crystallizability.

Recently, several PLLA-based nanotechnologies have emerged with an emphasis on achieving chemical, thermal, mechanical, and biological properties superior to conventional biopolymers, opening new possibilities for the plastic industry. However, since polylactic acid (PLA) is classified as a hard bio-polyester that is prone to hydrolysis, understanding and engineering of its thermo-mechanical properties and its nanocomposites are crucial for use in cutting-edge applications [5,6]. Along with many other interesting nanofillers, the use of layered transition-metal dichalcogenide nanostructures (TMDCs), such as molybdenum disulfide $\left(\mathrm{MoS}_{2}\right)$ and tungsten disulfide $\left(\mathrm{WS}_{2}\right)$, which are high-band gap semiconductors with $0 \mathrm{D}, 1 \mathrm{D}$, and 2D structural anisotropy are particular interesting. As an emerging 2D layered nanomaterial, it has been recently reported that monolayer $\mathrm{MoS}_{2}$ with high surface area, superb thermal stability, and excellent mechanical properties [7] exhibits great potential as a reinforcement agent for polymers [8,9]. In addition, it has been shown that the 2D-TMDCs can potentially improve the polymeric materials mechanical and barrier properties, whilst not effecting their electrical insulation properties (e.g., polyurethane (PU) [8], polypropylene (PP) [10], poly(vinyl alcohol) (PVA) [11]. In particular, the use of environmentally friendly and biocompatible inorganic TMDCs have been shown to offer design, processing, performance, and cost advantages compared to carbon nanotubes, nanoclays, or other inorganic nanoparticles $[12,13]$ when manufacturing advanced biopolymer nanocomposites (Bio-PNCs 1D-WS 2 ) (poly(propylene fumarate) (PPF), poly(3-hydroxybutyrate) (PHB), poly(ether ether ketone) (PEEK), PLLA, etc.) [14-17]. More specifically, the thermo-mechanical properties of PLLA biopolymers are directly related to their biomedical performance when interfaced with biological systems, since these properties can be used to optimize important design criteria (e.g., modulus, strength, morphology, crystallinity, biocompatibility, etc.) and, in turn, these properties can affect cell response, tissue regeneration, and in vivo degradation. In addition, research shows that different thermal treatments affect not only the crystallinity of PLLA polymers but also of other bio-polyesters, which is a critical parameter for cell biocompatibility and drug release dynamics [18].

It is well known that the crystallization of polymers is complex and is affected by a variety of factors that include temperature, cooling rate, and flow-induced deformation as well as the size, shape, and volume fraction of additive nanoparticles. In particular, controlling the crystallinity of hybrid polymeric systems has an important impact on their properties and is essential for developing novel functional materials. Despite many experiments to understand the effect of nano-additives on crystallization, the results have often been contradictory. Therefore, the control of crystallinity in hybrid molecular systems remains empirical at best. Jabbarzadeh has recently investigated the origins of enhanced and retarded crystallization in nanocomposite polymers [19]. The results of large-scale molecular dynamics simulations revealed that while crystallinity was affected by the nanoparticle size and its volume fraction, their combined effects can only be measured by interparticle free space and the characteristic size of the crystals. Understanding the dynamics of these systems, including the mobilities of the different constituents, also remains an extremely difficult task, despite the wide-ranging research interest in them $[20,21]$.

On the other hand, the study of biodegradability of biopolymer nanocomposite materials using laboratory-scale testing is extremely important from both an industrial and scientific perspective. Biodegradation can be influenced by many different factors, including biopolymer characteristics, the type of microoganism, and pre-treatment. Polymer characteristics, such as mobility, tacticity, crystallinity, molecular weight, chemical functionality, and substituents present in its structure, and plasticizers or nanoparticle additives added to the polymer all play an important role in its degradation. In addition, it has been reported [22,23] that adding hydrophilic nanoparticles can accelerate PLA biodegradation as water molecules can more easily penetrate into the polymeric matrix. However, other studies $[24,25]$ have reported that biodegradation was retarded due to an enhancement in the nanocomposites barrier properties. 
The aim of the current study is to demonstrate the advantages of using $2 \mathrm{D}-\mathrm{WS}_{2}$ as a suitable nano-reinforcement to enhance PLLA performance. The nanocomposites were prepared via a versatile, economic, and scalable melt-processing route. In particular, the influence of the $2 \mathrm{D}-\mathrm{WS}_{2}$ on the processability, morphology, biodegradation, and crystallization behavior of the resulting PLLA/2D-WS 2 nanocomposites are analyzed.

\section{Experimental Section}

\subsection{Materials and Processing}

PLLA in granule form (density $=1.25 \mathrm{~g} / \mathrm{cm}^{3}, M_{\mathrm{W}} \approx 1.5 \times 105 \mathrm{~g} / \mathrm{mol}$ ) was supplied by Goodfellow Ltd. (Huntingdon, UK) and used as received. The $2 \mathrm{D}-\mathrm{WS}_{2}$ nanosheets (density $\approx 7.5 \mathrm{~g} / \mathrm{cm}^{3}$, width/length $\approx$ 20-500 nm, and thickness $\approx 1 \mathrm{~nm}$ ) were obtained from ACS Material LLC (Medford, MA, US) and used without chemical modification. To prepare the PLLA/2D-WS ${ }_{2}$ nanocomposites, PLLA and 2D-WS $2(0.1$, 0.5 and $1.0 \mathrm{wt} \%$ ) were dispersed together in a small volume of ethanol (HPLC grade, Sigma-Aldrich Química SL, Madrid, Spain) and homogenized by mechanical stirring and bath ultrasonication for approximately $10 \mathrm{~min}$. Subsequently, the ethanol was evaporated off, and the PLLA/2D-WS 2 dispersion was dried under vacuum at $60{ }^{\circ} \mathrm{C}, 70 \mathrm{mbar}$ for $24 \mathrm{~h}$. Melt-mixing of the resulting dispersions was performed using a micro-extruder (Thermo-Haake Minilab system) operated at $190{ }^{\circ} \mathrm{C}$ and a rotor speed of $100 \mathrm{rpm}$ for $10 \mathrm{~min}$ [17]. Then, the samples were pressed into film thicknesses of $0.3-0.5 \mathrm{~mm}$ in a hot press system using two heating/cooling plates (Collin P-200, Collin Lab \& Pilot Solutions GmbH, Maitenbeth, Germany).

\subsection{Characterization Studies}

\subsubsection{Scanning Electron Microscopy (SEM)}

The morphology of degradable and non-degradable samples was characterized using ultra-high field-emission scanning microscopes (FESEM), JEOL-JSM7600F, and SU8000-Hitachi Co., Ltd. (Tokyo, Japan), respectively. All specimens were sputter coated with gold or/and $\mathrm{Au} / \mathrm{Pd}$ prior to analysis.

\subsubsection{Wide-Angle $X$-ray Diffraction (WAXS)}

WAXS diffractograms were obtained using a Bruker D8 Advance diffractometer (Bruker AXS $\mathrm{GmbH}$, Karlsruhe, Germany) employing Ni-filtered CuK $\alpha$ radiation $(\lambda=0.15418 \mathrm{~nm})$ over the angular region $2 \theta$ between $5^{\circ}$ and $40^{\circ}$. Compression-molded film samples were crystallized from the melt at $220^{\circ} \mathrm{C}$ at cooling rates of $5^{\circ} \mathrm{C} / \mathrm{min}$ in a Mettler FP90/FP82 HT temperature cell (Mettler-Toledo SAE, Barcelona, Spain).

\subsubsection{Differential Scanning Calorimetry (DSC)}

The non-isothermal crystallization studies were carried out using a Perkin Elmer DSC7/7700 differential scanning calorimeter (Perkin-Elmer España SL, Madrid, Spain) under a nitrogen purge. The instrument was calibrated for temperature and heat flow using high-purity indium and zinc standards, and the data were evaluated by using the DSC-7/UNIX program. A tau lag calibration of the instrument for different heating rates was performed using indium. The experimental and theoretical procedures used in this study were similar to those employed in our previous publication on PLLA/1D-WS 2 [17]. The samples were first heated to $225^{\circ} \mathrm{C}$ and held at this temperature for $5 \mathrm{~min}$ to erase their thermal history. Afterwards, cooling cycles from the melt were then undertaken for each sample at cooling rates $(\varphi)$ of $1,2,5,10$ and $20^{\circ} \mathrm{C} / \mathrm{min}$. The heat that evolved during the non-isothermal crystallization was recorded as a function of temperature. The crystallization peak temperature $\left(T_{p}\right)$ was determined from the minimum of the crystallization exotherm observed during the cooling scan. The apparent crystallization enthalpy was determined as the area below the transformation curve, 
taking as the upper and lower limits as the corresponding deviations in the baseline, crystallinity was calculated as follows:

$$
(1-\lambda)=\frac{\Delta H_{c}}{\Delta H_{m}^{0}}
$$

where $\Delta H_{c}$ is the crystallization enthalpy and $\Delta H_{m}^{0}$ is the enthalpy of melting for perfect crystals $(93 \mathrm{~J} / \mathrm{g})[26]$.

\subsubsection{Biodegradation Tests}

The bacterial degradation of PLLA and the PLLA/2D-WS2 nanocomposite films was performed using the actinobacteria, Lentzea waywayandensis (DSM 44232) obtained from DSMZ-German Collection of Microorganisms and Cell Cultures $\mathrm{GmbH}$. For this, sterilized films ( $5 \mathrm{~mm} \times 5 \mathrm{~mm} \times 0.3 \mathrm{~mm}$ ) were placed in Erlenmeyer flasks containing $90 \mathrm{~mL}$ of basal culture medium that were supplemented with $0.1 \%$ gelatin and $10 \mathrm{~mL}$ of the actinobacteria liquid culture prepared according to reported procedures [27]. The biodegradation tests of the nanocomposite films were carried out at $30{ }^{\circ} \mathrm{C}$ and $180 \mathrm{rpm}$ using an orbital shaker (KS $4000 \mathrm{i}$ control, IKA) for incubation periods of 7, 14 and 21 days. All the tests were carried out in duplicate with control tests also conducted in the absence of the microorganism.

\section{Results}

\subsection{Morphology and Structure}

It is well known that the dispersion and interfacial interaction between nanofillers and biopolymer matrices play a key role in the final properties of biopolymer nanocomposites [17]. SEM was employed to observe the micromorphology of the cryogenically fractured surfaces of PLLA, the nanocomposite films, and the neat $\mathrm{WS}_{2}$ nanosheets (Figure 1).
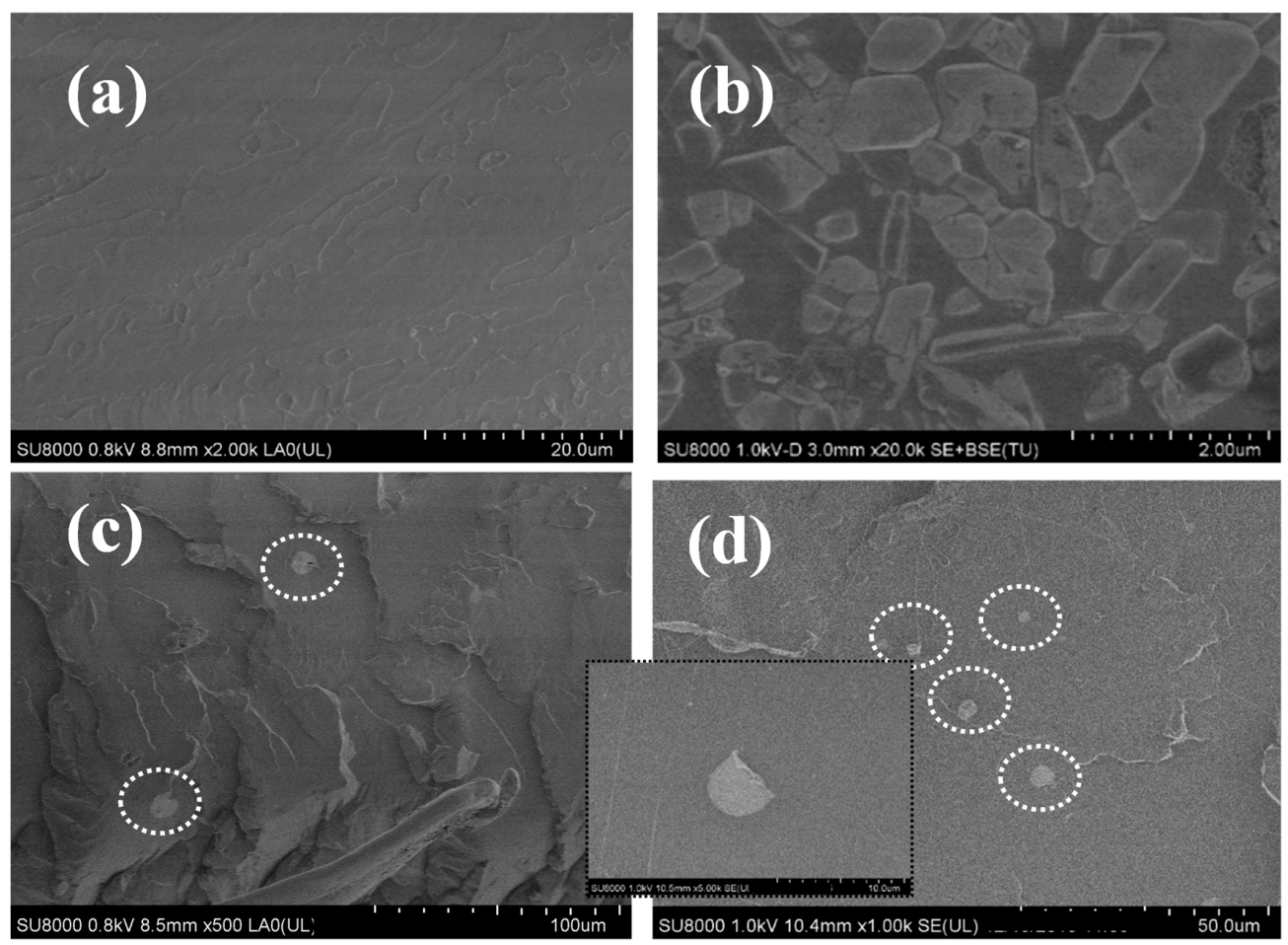

Figure 1. SEM micrographs of (a) poly(L-lactic acid) (PLLA), (b) tungsten disulfide nanosheets $\left(2 \mathrm{D}-\mathrm{WS}_{2}\right)$ and PLLA/2D-WS 2 nanocomposites with nanofiller loadings of (c) 0.5 and (d) $1.0 \mathrm{wt} \%$. The white dashed circles represented the $2 \mathrm{D}-\mathrm{WS}_{2}$. 
The morphological differences between PLLA and the PLLA/2D-WS ${ }_{2}$ nanocomposites are clearly visible. From Figure 1a, it can be seen that the fractured PLLA surface is comparatively smooth. In contrast, the fracture surfaces of the PLLA/2D-WS ${ }_{2}$ nanocomposites (Figure 1c,d) are relatively rough with the $\mathrm{WS}_{2}$ nanosheets being well dispersed, and are neither fully enclosed nor pulled-out from the PLLA matrix. This suggests that there is a strong interfacial interaction between the 2D-WS 2 and the PLLA matrix encountered using simple shear force melt-blending. Typically, to achieve this, more elaborate methodologies have been employed, such as the synthesis of PLA/MoS $2-\mathrm{NH}_{2}$ nanocomposites via in situ ring-opening polymerization [28].

Wide-angle X-ray diffraction (WAXD) measurements were performed on the PLLA/2D-WS nanocomposites film samples with the same thermal history to be able to determine whether the addition of $2 \mathrm{D}-\mathrm{WS}_{2}$ affected the PLLA crystalline structure (Figure 2). At room temperature, only the characteristic diffraction peaks of PLLA are seen, with the strongest visible diffraction peak being the characteristic (200)/(110) reflection of the $\alpha$-form at $16.7^{\circ}$ [17], implying that the $2 \mathrm{D}-\mathrm{WS}_{2}$ nanoparticles have no impact on its crystalline structure. However, the crystallite size perpendicular to the diffraction characteristic (200)/(110) plane, $\mathrm{D}_{200 / 110}$, obtained from the room temperature diffractograms using well-known Scherrer formula, increases with the addition of 2D-WS ${ }_{2}(P L L A=28.6 \mathrm{~nm}$, PLLA/2D-WS $(0.1 \mathrm{wt} \%)=37.0 \mathrm{~nm}, \mathrm{PLLA} / 2 \mathrm{D}-\mathrm{WS}_{2} 0.5 \mathrm{wt} \%=33.1$ and PLLA/2D-WS $\left.2(1.0 \mathrm{wt} \%)=29.9 \mathrm{~nm}\right)$. In particular, during the cooling process, PLLA crystals grow considerably, resulting in larger room temperature $\mathrm{D}_{200 / 110}$ values than the calculated for the pure matrix. On the other hand, the disappearance of the layered transition-metal dichalcogenide diffraction peaks suggests that the nanoparticle is highly exfoliated and/or the PLLA is well intercalated within the $\mathrm{WS}_{2}$ sheets $[9,10]$.

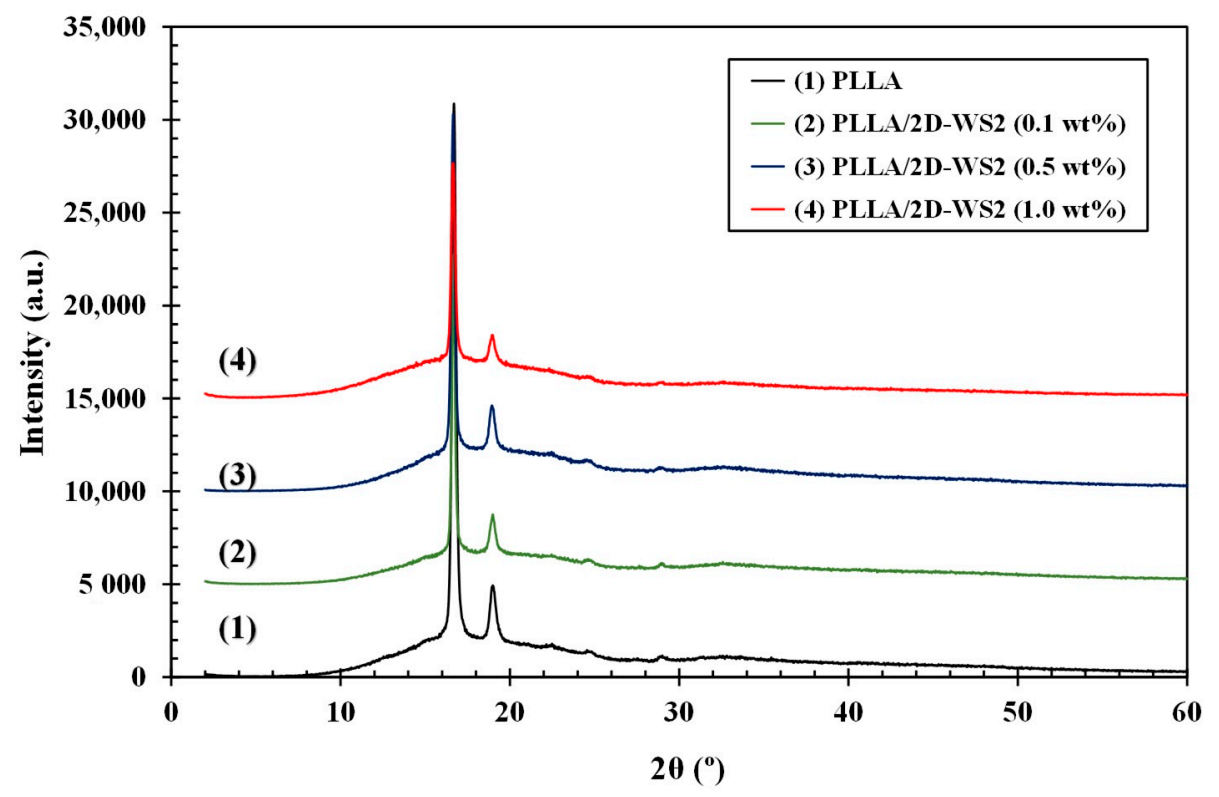

Figure 2. WAXS diffractograms of PLLA/2D-WS 2 nanocomposites obtained at room temperature after cooling from the melt at $5{ }^{\circ} \mathrm{C} / \mathrm{min}$.

\subsection{Non-Isothermal Crystallization}

The non-isothermal crystallization behavior of PLLA and the PLLA/2D-WS ${ }_{2}$ nanocomposites was investigated as this corresponds to the type of temperature changes that might occur in industrial applications. Figure 3 shows the effect of cooling rate and $2 \mathrm{D}-\mathrm{WS}_{2}$ concentration on the non-isothermal crystallization behavior of the PLLA/2D-WS 2 nanocomposites with the specific crystalline parameters of all samples listed in Table 1. 

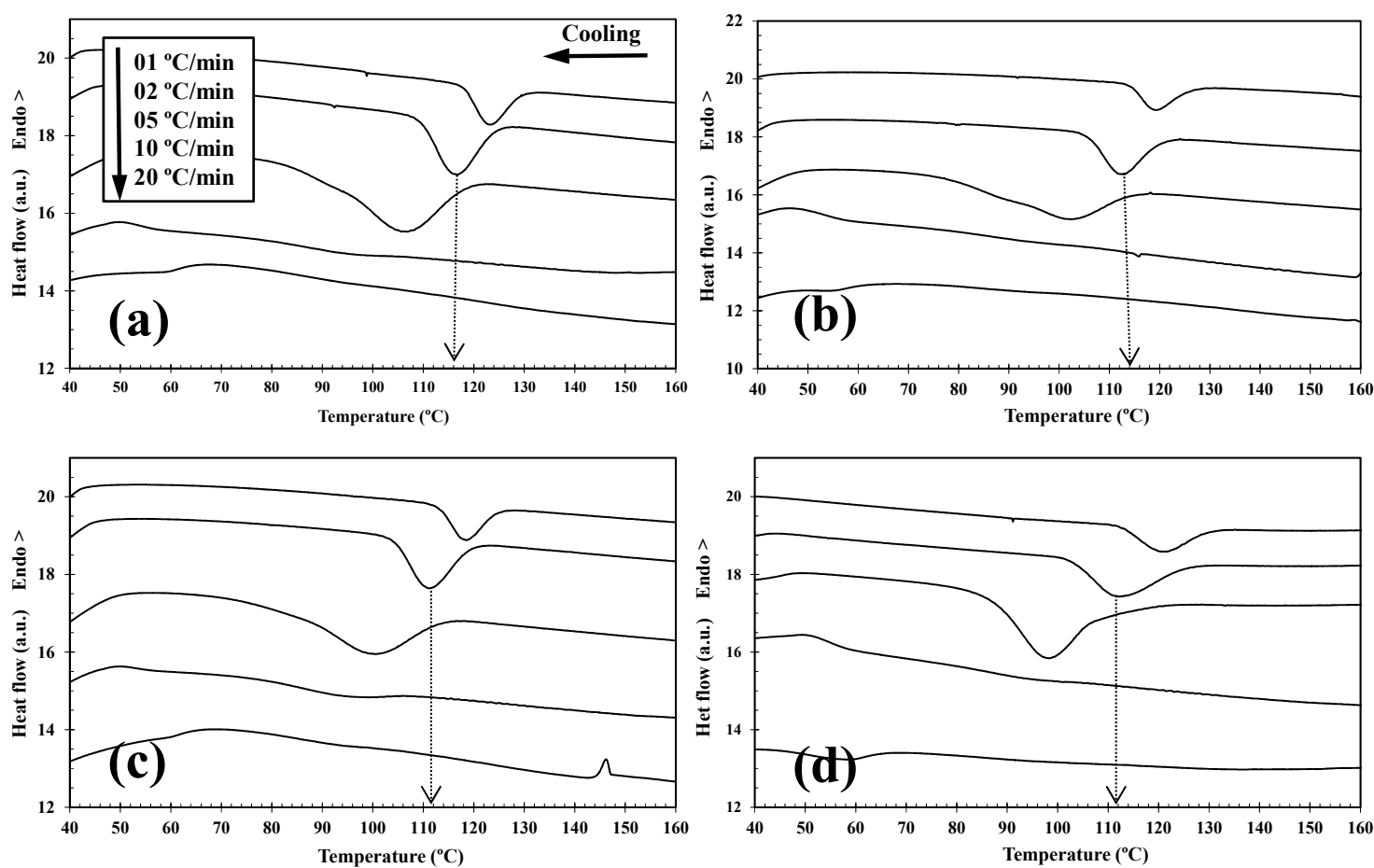

Figure 3. Differential Scanning Calorimetry (DSC) melt-crystallization thermograms of (a) PLLA and PLLA/2D-WS 2 nanocomposites with nanofiller loadings of (b) 0.1 , (c) 0.5 , and (d) $1.0 \mathrm{wt} \%$ obtained at the cooling rates indicated.

Table 1. Crystallization parameters of the PLLA/2D-WS 2 nanocomposites.

\begin{tabular}{|c|c|c|c|c|c|c|c|}
\hline $2 \mathrm{D}-\mathrm{WS}_{2}$ & $\begin{array}{c}\varphi \\
\left({ }^{\circ} \mathrm{C} / \mathrm{min}\right)\end{array}$ & $\begin{array}{c}T_{p} \\
\left({ }^{\circ} \mathrm{C}\right)\end{array}$ & $\begin{array}{c}(1-\lambda)^{c} \\
(\%)\end{array}$ & $\begin{array}{l}x^{a} \\
(\%)\end{array}$ & $\alpha^{b}$ & $f(T)^{\mathbf{b}}$ & $\begin{array}{c}\Delta E^{\mathrm{c}} \\
(\mathrm{kJ} / \mathrm{mol})\end{array}$ \\
\hline \multirow{5}{*}{0} & 1 & 123.1 & 57.7 & 10 & 1.09 & 5.01 & \multirow{5}{*}{-119.9} \\
\hline & 2 & 116.5 & 53.2 & 30 & 1.11 & 5.1 & \\
\hline & 5 & 105.6 & 45.9 & 50 & 1.12 & 5.18 & \\
\hline & 10 & 94.3 & 4 & 70 & 1.14 & 5.27 & \\
\hline & 20 & - & - & 90 & 1.17 & 5.45 & \\
\hline \multirow{5}{*}{0.1} & 1 & 119.3 & 56.1 & 10 & 1.1 & 5.08 & \multirow{5}{*}{-114.1} \\
\hline & 2 & 112.1 & 51.4 & 30 & 1.11 & 5.13 & \\
\hline & 5 & 101.2 & 40.9 & 50 & 1.13 & 5.24 & \\
\hline & 10 & - & - & 70 & 1.15 & 5.35 & \\
\hline & 20 & - & - & 90 & 1.18 & 5.5 & \\
\hline \multirow{5}{*}{0.5} & 1 & 118.6 & 55.7 & 10 & 1.09 & 5.03 & \multirow{5}{*}{-118.2} \\
\hline & 2 & 110.9 & 51.1 & 30 & 1.1 & 5.1 & \\
\hline & 5 & 99.4 & 36.9 & 50 & 1.1 & 5.16 & \\
\hline & 10 & 93.4 & 3.5 & 70 & 1.11 & 5.21 & \\
\hline & 20 & - & - & 90 & 1.13 & 5.31 & \\
\hline \multirow{5}{*}{1} & 1 & 120.7 & 57.6 & 10 & 1.09 & 5.01 & \multirow{5}{*}{-99.9} \\
\hline & 2 & 112.1 & 56.2 & 30 & 1.11 & 5.11 & \\
\hline & 5 & 98.2 & 45.7 & 50 & 1.11 & 5.17 & \\
\hline & 10 & - & 3 & 70 & 1.12 & 5.22 & \\
\hline & 20 & - & - & 90 & 1.12 & 5.28 & \\
\hline
\end{tabular}

a The corresponding values of volume fraction are $0.01,0.13$, and $0.67 \%{ }^{b}$ Crystallization parameters calculated using Liu's equation. ${ }^{\mathrm{c}}$ Effective energy barrier calculated using Kissinger's equation.

From the previous curves, useful parameters, such as the peak temperature $\left(T_{p}\right)$ and crystallinity $(1-\lambda)_{\mathrm{c}}$ as a function of crystallization temperature, can be obtained to describe the non-isothermal crystallization behavior of the tested materials. It can be seen that PLLA manifests slow crystallization 
on cooling from the melt, and it does not crystallize at a cooling rate of $10^{\circ} \mathrm{C} / \mathrm{min}$ or faster. Additionally, as the cooling rate increases, the crystallization exotherm broadens and shifts to lower temperatures for both the PLLA and PLLA/2D-WS ${ }_{2}$. This indicates that at slower cooling rates, a larger proportion of the tested semicrystalline polymers spent more time within a temperature range sufficient to promote chain segment mobility and crystal growth. With the increase in the cooling rate, the crystallization of the composite material gradually decreased. This is because the frozen molecular chain segments prevented the crystallization of PLA when the cooling rate was too fast. Furthermore, for a given cooling rate, the $T_{p}$ of PLLA/2D-WS ${ }_{2}$ was lower than that of pure PLLA, as shown in Figure 3, indicating that the addition of $2 \mathrm{D}-\mathrm{WS}_{2}$ into PLLA decreased its rate of crystallization. This is because the surface of the $\mathrm{WS}_{2}$ nanosheets could not easily adsorb the PLLA chain segments, which would greatly hinder crystal growth. In particular, when the interparticle free space becomes smaller than the characteristic extended length of the polymer molecule, nanoparticles impede crystallization due to confinement effects. Based on the findings from the work of Jabbarzadeh, equations for critical particle size or volume fraction that led to this confinement-induced retardation of crystallization were proposed [19].

For more clarity, Figure 4 summarizes the variation of $T_{p}$ with cooling rate and composition. In particular, as the addition of $2 \mathrm{D}-\mathrm{WS}_{2}$ reduces the crystallization temperature of PLLA, it would imply that the nucleation of PLLA crystals is retarded by the $\mathrm{WS}_{2}$ nanosheets. This observation is reproducible for nanocomposites crystallized at different cooling rates. In contrast, $1 \mathrm{D}-\mathrm{WS}_{2}$ nanotubes have been shown to accelerate the PLLA crystallization process via heterogeneous nucleation [17]. Such differences suggest that the nanoparticle shape plays a fundamental role in PLLA crystallization. In a similar manner to the $2 \mathrm{D}-\mathrm{WS}_{2}$ nanosheets, the addition of Cloisite 30B (a organically modified montmorillonite [28]) to PLA was also found to retard its crystallization process. This was reported to be due to the good interfacial energy between the PLA matrix and the modifier used in Cloisite 30B hindering the PLA chain-folding process needed for crystallization. As such, it suggests that highly compatible clays dispersed within the polymer matrix can hinder the interchain interactions necessary for crystal nuclei formation. This discrepancy is likely related to several factors, including the difference in the thermal conductivity of the filler and polymer matrix, the nucleation efficiency (NE) of the filler, its state of dispersion within the matrix, and the potential existence of mechanisms of interfacial crystallization such as epitaxy and transcrystallization [29-32]. NE is strongly dependent on the nanofiller morphology, its surface energy, roughness, and crystalline structure as well as on the filler ability to form the critical nucleus $[16,17,33,34]$. Furthermore, the dependence of crystallinity $(1-\lambda)_{c}$ of PLLA and its $2 \mathrm{D}-\mathrm{WS}_{2}$ nanocomposites as a function of cooling rate (Figure $5 \mathrm{a}$ ) closely mirrors the $T_{p}$ trends previously mentioned. This is expected, as at slower cooling rates, the polymer chains have more time to organize into crystalline domains with fewer defects and thus will present a higher $(1-\lambda)_{c}$. However, the crystallinity value obtained from the crystallization exotherm of PLLA appears unchanged with the addition of $2 \mathrm{D}-\mathrm{WS}_{2}$, particularly at low cooling rates (Figure $5 \mathrm{~b}$ ). 

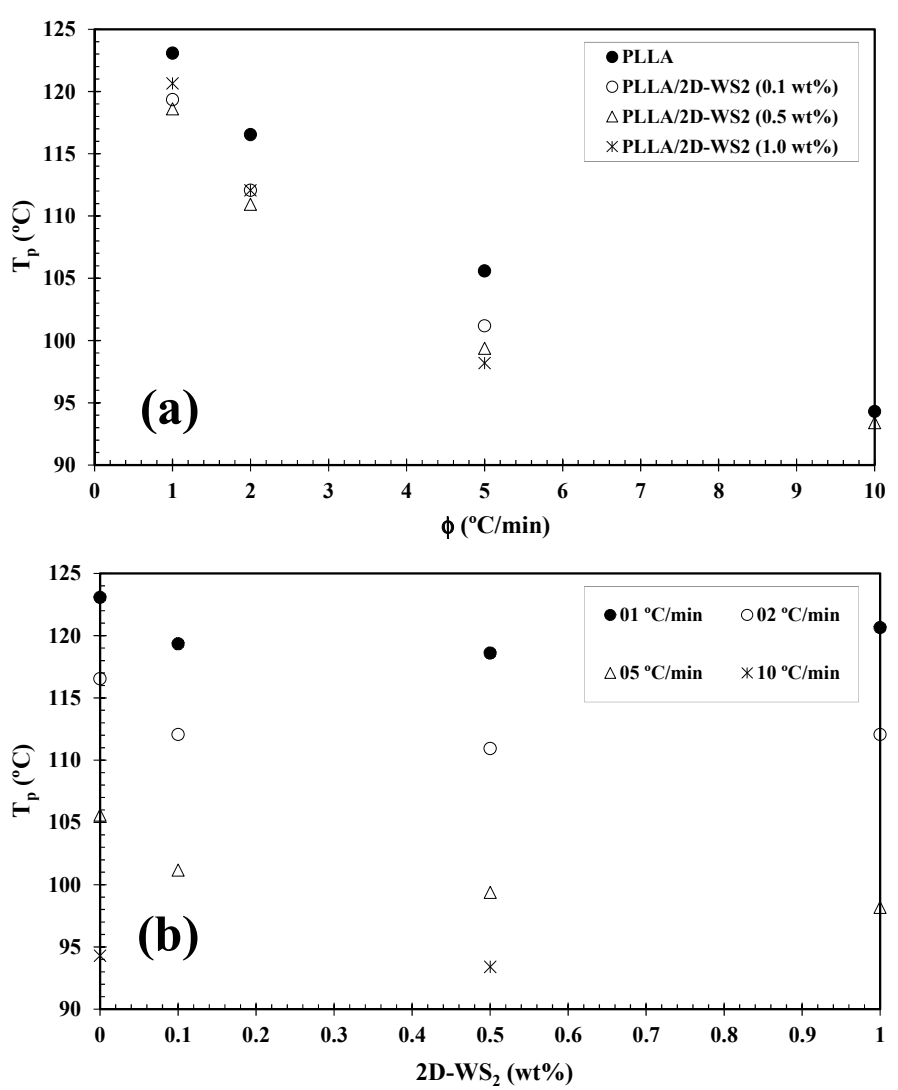

Figure 4. Variation of the crystallization peak temperature $\left(T_{p}\right)$ for PLLA/2D-WS $\mathrm{W}_{2}$ nanocomposites with (a) cooling rate and (b) composition.
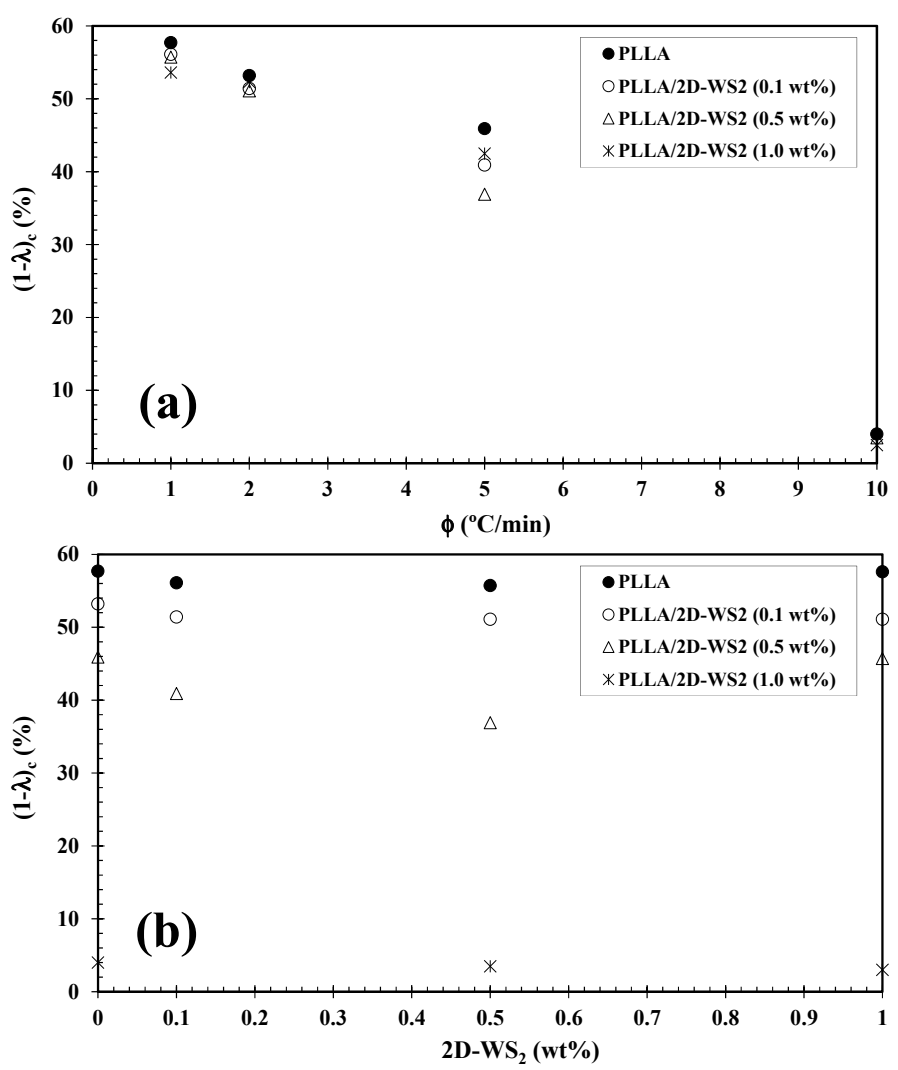

Figure 5. Variation of the crystallinity $(1-\alpha)_{c}$ for PLLA/2D-WS 2 nanocomposites with (a) cooling rate and (b) composition. 
It is well-known that polymer crystallization releases a significant amount of heat, making DSC the preferred method for measuring overall crystallization kinetics. The measured rate of heat release is assumed to be proportional to the macroscopic rate of crystallization:

$$
\frac{d Q}{d t}=Q_{c} \frac{d x}{d t}
$$

where $Q_{c}$ is the measured heat of crystallization calculated by integration of the DSC peak. The values of $Q_{c}$ can further be used to determine the crystallization rate $(d x / d t)$ as well as the extent of the melt conversion:

$$
x(t)=\frac{1}{Q_{c}} \int_{0}^{t} \frac{d Q}{d t} d t
$$

The value of $x(t)$ varies from 0 to 1 and represents the degree of conversion. The transformation from temperature to time is performed using a constant cooling rate $\varphi$ :

$$
t=\frac{T_{0}-T}{\varphi}
$$

where $T$ is the temperature at time $t$ and $T_{\mathrm{i}}$ is the temperature at the start of crystallization. Figure 6 shows typical conversion curves at various cooling rates for the PLLA/2D-WS ${ }_{2}$ nanocomposites. The conversion curves shift over to longer times with decreasing cooling rates, suggesting that the diffusion of PLLA becomes very difficult for melt crystallization. In order to quantitatively describe the evolution of crystallinity during non-isothermal crystallization, a number of models have been proposed in the literature. In this investigation, the Lui model was tested.
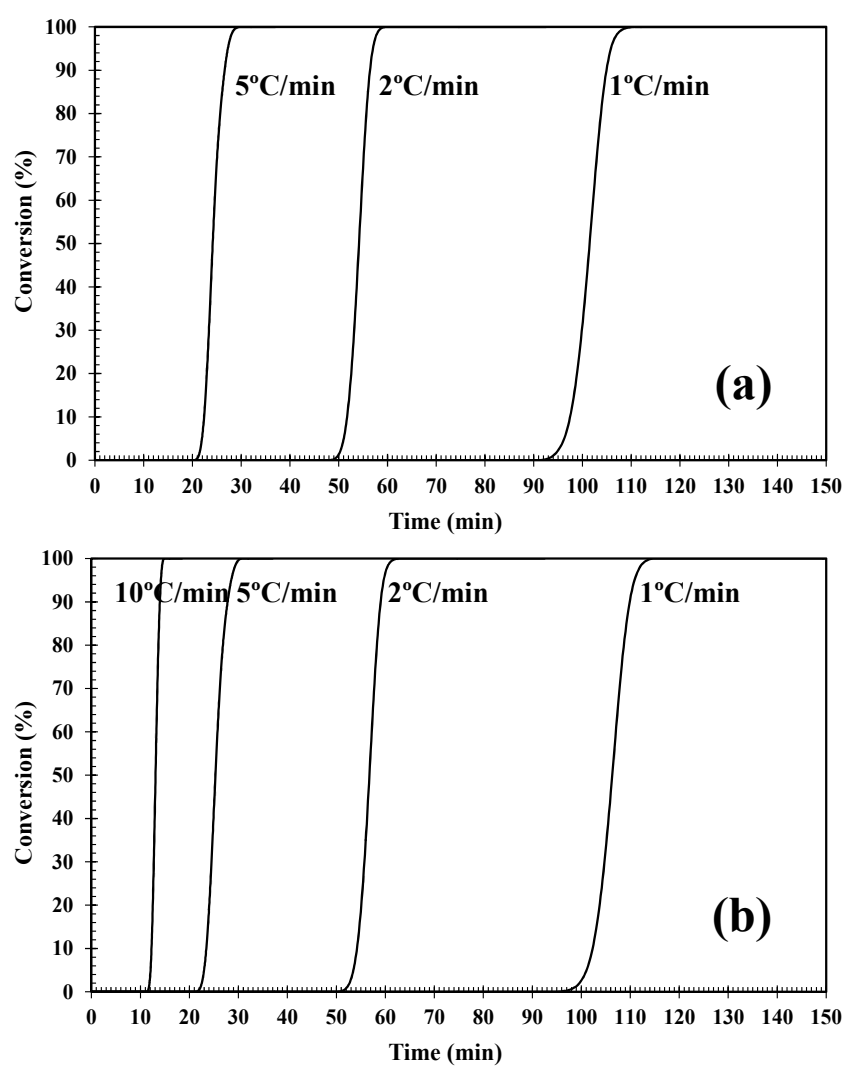

Figure 6. Plots of degree of conversion (x) vs. time for (a) PLLA and (b) PLLA/2D-WS 2 (0.5 wt \%) crystallized non-isothermally at various cooling rates. 


\subsection{Lui Model}

A convenient approach adopted to describe the non-isothermal crystallization was the Liu model [35]. By combining the Avrami [36-38] and Ozawa [39] equations, the Liu model has been proved to be suitable and convenient to handle the non-isothermal crystallization of polymer nanocomposites [40]. As the degree of conversion $(x)$ is related to the cooling rate $\varphi$ and the crystallization time $t$ (or temperature $T$ ), the relation between $\varphi$ and $t$ could be defined for a given degree of conversion. Consequently, the kinetic equation for non-isothermal crystallization was derived:

$$
\ln \varphi=\ln f(T)-\alpha \ln t
$$

where $f(T)=\left[\mathrm{k}^{\prime}(\mathrm{T}) / \mathrm{k}\right]^{1 / \mathrm{m}}$ refers to the value of cooling rate chosen at a unit crystallization time, when the system has a certain degree of crystallinity, and $\alpha$ is the ratio of the Avrami exponents to Ozawa exponents (i.e., $\alpha=n / m$ ). According to Equation (5), at a given degree of conversion, the plot of $\ln \varphi$ vs. $\ln t$ gives a series of lines, as can be seen in Figure 7. This indicates that the Lui model provides a satisfactory description for the non-isothermal crystallization for PLLA/2D-WS ${ }_{2}$ nanocomposites. The kinetic parameters, $\ln f(T)$ and $\alpha$, which are derived from the slope and the intercept of those lines respectively, are listed in Table 1.
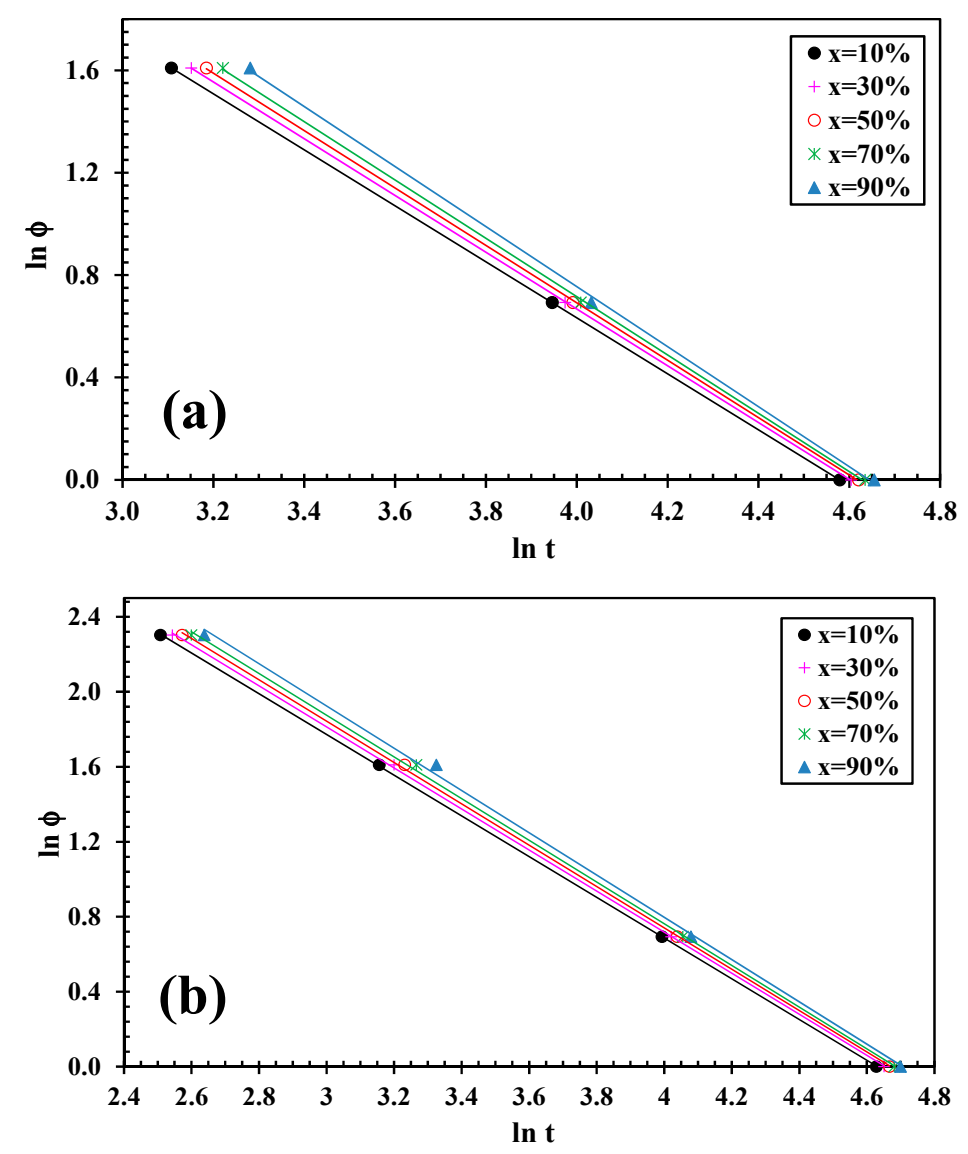

Figure 7. Lui plots for melt crystallization of (a) PLLA and (b) PLLA/2D-WS 2 ( $0.5 \mathrm{wt} \%)$.

The $f(T)$ values increased rapidly with an increase in the relative crystallinity of all samples. However, the $f(T)$ value of PLLA/2D-WS ${ }_{2}$ is smaller than that of neat PLLA, meaning that the addition of $2 \mathrm{D}-\mathrm{WS}_{2}$ to PLLA needs a higher cooling rate to approach an identical degree of crystalline transformation. In other words, the rate of crystallization of the PLLA/2D-WS ${ }_{2}$ nanocomposites is lower than that of PLLA. This is also in good agreement with the results observed in Figures 3 and $4 \mathrm{~b}$. In addition, the values of the parameter $\alpha$ are nearly constant (1.1 to 1.7$)$, indicating 
that the mechanism of nucleation and growth is approximately the same for both PLLA and the PLLA/2D-WS ${ }_{2}$ nanocomposites.

\subsection{Effective Energy Barrier}

There are many mathematical approaches to evaluate the crystallization activation energy, or effective energy barrier, $\Delta E$ of the crystallization process. The approach proposed by Kissinger is used in this study [41]. Considering the variation of the crystallization peak temperature $T_{p}$ with cooling rate $\varphi$, the $\Delta E$ could be determined as follows:

$$
\ln \left(\frac{\varphi}{T_{p}^{2}}\right)=\text { Constant }-\frac{\Delta E}{R T_{p}}
$$

where $R$ is the universal gas constant. The calculated values of activation energy (Figure 8) are given in Table 1. It can be concluded that the addition of $2 \mathrm{D}-\mathrm{WS}_{2}$ caused a decrease in the $\Delta E$, making the molecular chains of PLLA more difficult to crystallize. As such, it is verified again that the $2 \mathrm{D}-\mathrm{WS}_{2}$ do not nucleate PLLA.

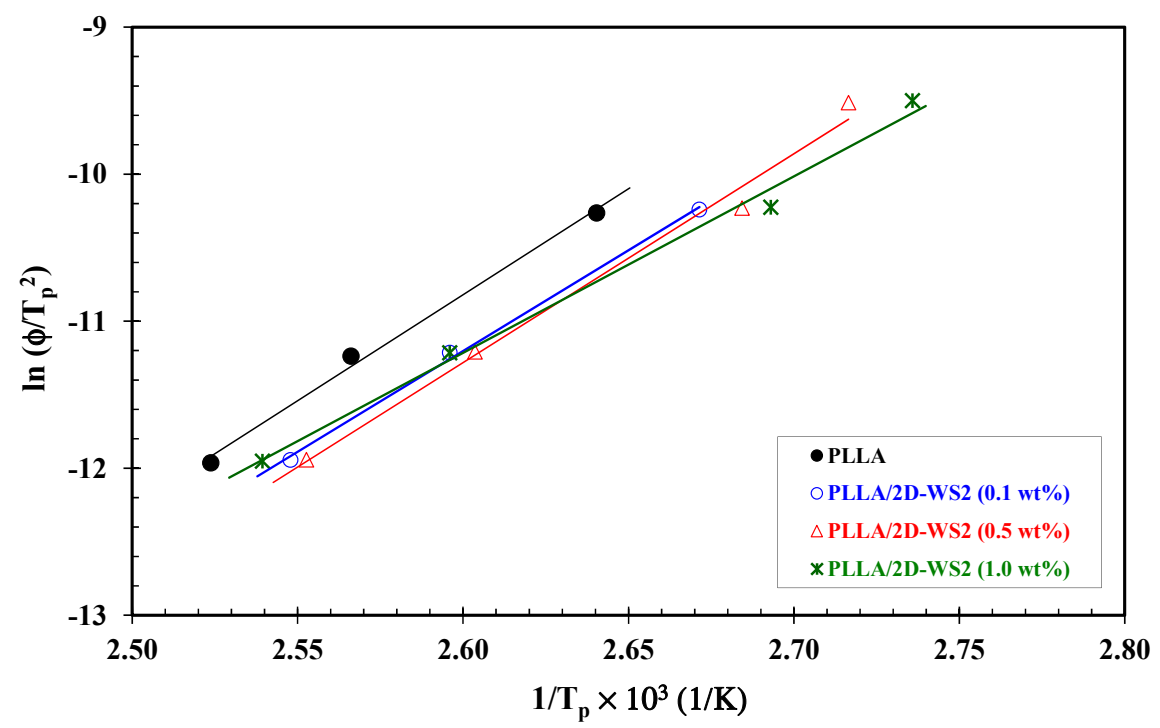

Figure 8. Kissinger plots for evaluating effective energy barrier of PLLA/2D-WS ${ }_{2}$ nanocomposites.

\subsection{Biodegradation Tests}

Polymer degradation is associated with changes in characteristics, such as the color and surface morphology. Effects used to describe degradation include roughening of the surface, the formation of holes or cracks, de-fragmentation, changes in color, or the formation of bio-films on the surface. The PLLA films, which were initially transparent and amorphous, became a translucent white after 7 days of incubation in the presence of Lentzea waywayandensis. After 21 days, the surface of the neat PLLA changed to a yellowish-dark brown color, which is caused by water permeation and microorganism activity. Figure 9 shows the surface morphology of PLLA and the 2D-WS 2 nanocomposite films under SEM. Before the degradation trials, the surface of neat PLLA and the PLLA/2D-WS 2 nanocomposites was smooth. After 7 days, the neat PLLA did not present any significant surface changes in the presence of the actinobacteria, and at 14 days, only the surface roughness had increased. However, in the case of the PLLA/2D-WS 2 nanocomposites after 7 days, their surfaces exhibited the presence of obvious cracks and clearly showed considerable degradation likely as a result of enhanced PLLA hydrolysis and microorganisms activity. With the addition of $\mathrm{WS}_{2}$ nanosheets, the cracks and voids became substantially deeper and larger and thereby suggest more surface erosion 
during a shorter incubation period. This bulk erosion hydrolytic degradation process is comparable to that observed for PLA and PLA $/ \mathrm{T}_{\mathrm{i}} \mathrm{O}_{2}$ nanocomposite systems [19].

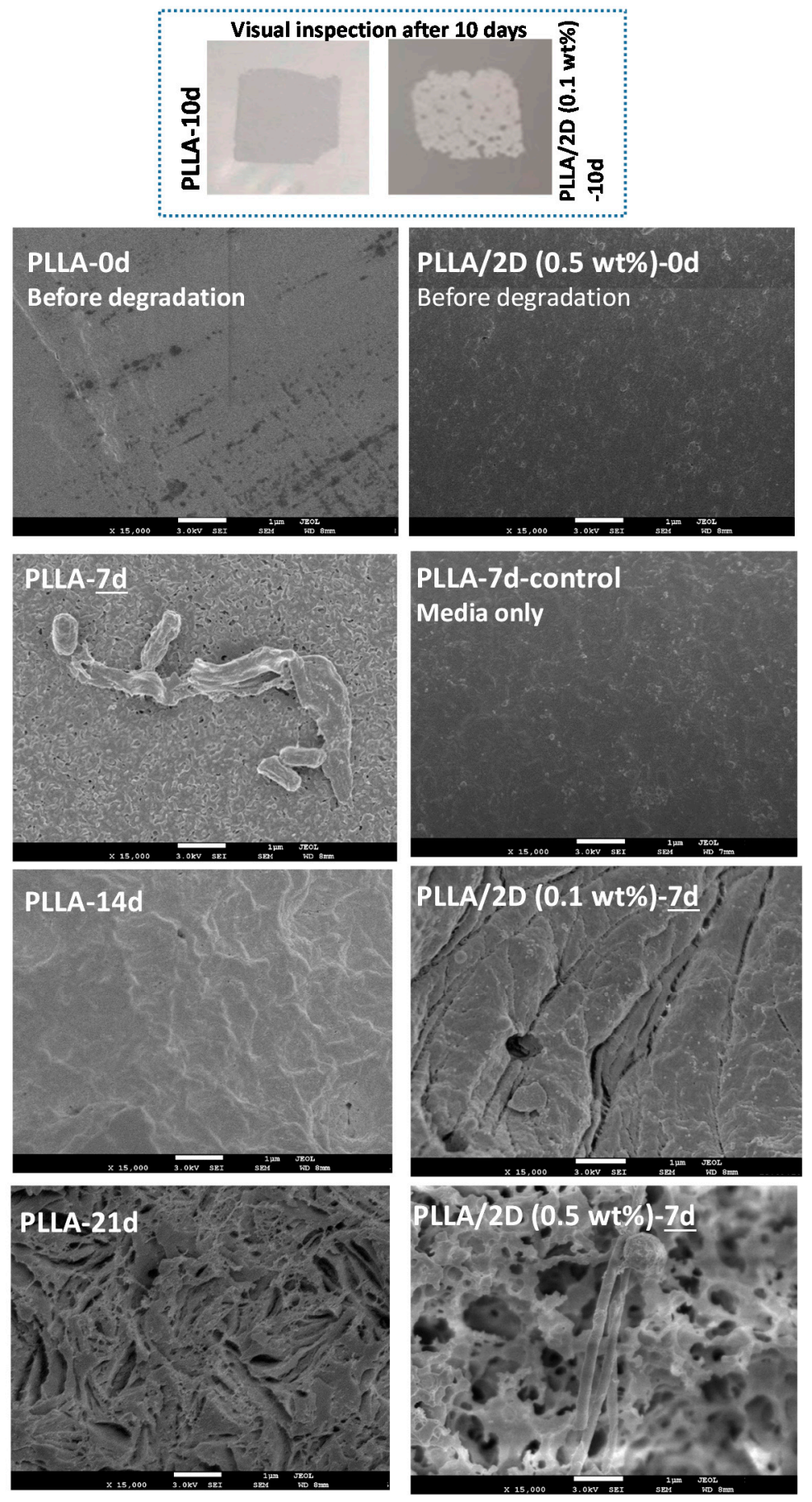

Figure 9. Optical and SEM micrographs of PLLA and PLLA/2D-WS 2 ( 0.5 and $1.0 \mathrm{wt} \%$ ) nanocomposites obtained at the incubation times indicated.

In summary, this study is the first step to exploring PLLA nanocomposites degradation using a promising actinobacteria (Lentzea waywayandensis) and understanding how degradation changes based on the addition of $2 \mathrm{D}-\mathrm{WS}_{2}$. In future work, we will focus on both the regulatory mechanisms involved in actinobacteria PLLA degradation as well as the enzymes acting upon the polymer. The combination of physical, chemical, and biochemical modifications to the active enzymes together with controlling regulatory mechanisms could lead to more efficient polymer degradation. 


\section{Conclusions}

In this work, the dispersion of $\mathrm{WS}_{2}$ nanosheets in a PLLA matrix was achieved via melt processing, a simple, scalable, cost-effective and ecologically method. SEM and WAXS demonstrated that the 2D-WS 2 were well dispersed, intercalated, and/or exfoliated in the PLLA matrix. DSC analysis revealed that the non-isothermal crystallization behavior of the PLLA/2D-WS ${ }_{2}$ nanocomposites was strongly dependent on the $2 \mathrm{D}-\mathrm{WS}_{2}$ content and cooling rate. In particular, the incorporation of $2 \mathrm{D}-\mathrm{WS}_{2}$ at a relatively low concentration induced a significant reduction in the crystallization rate of PLLA due to the physical barrier action of the nanosheets while maintaining the crystal structure of PLLA. Furthermore, the method developed by Liu at al. could successfully describe the complex crystallization kinetics of the PLLA/2D-WS 2 nanocomposites occurring during continuous cooling. The parameter $f(T)$, which has a physical and practical significance, decreased with $2 \mathrm{D}-\mathrm{WS}_{2}$ loading, indicating that the addition of the nanoparticles hindered the PLLA polymer chain transportation to the crystal growth front. In the same manner, the effective energy barrier governing the non-isothermal crystallization confirms the evident decrease in the PLLA crystallization rate in the PLLA/2D-WS $\mathrm{W}_{2}$ nanocomposites. Finally, biodegradation analysis showed that the incorporation of the $2 \mathrm{D}-\mathrm{WS}_{2}$ nanoparticles into the typically poorly biodegradable PLLA matrix facilitated its degradation in the presence of actinobacteria (Lentzea waywayandensis).

Author Contributions: M.N. and M.F. conceived and designed the work; A.M.G. and P.S.S. prepared the samples and performed the DSC experiments; A.M.G. prepared the bacterial cultures and materials for enzymatic biodegradation. D.A.M. performed the degradation tests; all authors contributed to the scientific discussion, and M.N. and P.S.S. wrote the paper. All authors have read and agreed to the published version of the manuscript.

Funding: This research was funded by FEDER/Ministerio de Ciencia, Innovación y Universidades-Agencia and Subprograma Estatal de Incorporación/IED2019-001134-I.

Acknowledgments: P.S.S. also acknowledges the Spanish Ministry of Economy, Industry, and Competitiveness (MINECO) for support through a Ramon y Cajal Fellowship (RYC-2014-16759). The authors appreciate the assistance and support of the ICTP-CSIC characterisation services.

Conflicts of Interest: The authors declare no conflict of interest.

\section{References}

1. Drumright, R.E.; Gruber, P.R.; Henton, D.E. Polylactic acid technology. Adv. Mater. 2000, 12, 1841-1846. [CrossRef]

2. Sinclair, R.G. The case for the polylactic acid as a commodity packaging plastic. Pure Appl. Chem. A 1996, 33, 585-597. [CrossRef]

3. Lim, L.T.; Auras, R.; Rubino, M. Processing technologies for poly(lactic acid). Prog. Polym. Sci. 2008, 33, 820-852. [CrossRef]

4. Rhim, J.W.; Mohanty, A.K.; Singh, S.P.; Ng, P.K.W. Effect of the processing methods on the performance of polylactide films: Thermocompression versus solvent casting. J. Appl. Polym. Sci. 2006, 101, 3736-3742. [CrossRef]

5. Bayer, I.S. Thermomechanical properties of polylactic acid-graphene composites: A state-of-the-art review for biomedical applications. Materials 2017, 10, 748. [CrossRef]

6. Casalini, T.; Rossi, F.; Castrovinci, A.; Perale, G. A perspective on polylactic acid-based polymers use for nanoparticles synthesis and applications. Front. Bioeng. Biotechnol. 2019, 7, 259. [CrossRef]

7. Rao, C.N.R.; Maitra, U.; Waghmare, U.V. Extraordinary attributes of 2-dimensional $\mathrm{MoS}_{2}$ nanosheets. Chem. Phys. Lett. 2014, 609, 172-183. [CrossRef]

8. Wang, X.; Xing, W.; Feng, X.; Yu, B.; Song, L.; Yeoh, G.H.; Hu, Y. Enhanced mechanical and barrier properties of polyurethane nanocomposite films with randomly distributed molybdenum disulfide nanosheets. Compos. Sci. Technol. 2016, 127, 142-148. [CrossRef]

9. Chen, P.; Liang, X.; Xu, Y.; Zhou, Y.; Nie, W. Enhanced thermal and mechanical properties of PLA/MoS 2 nanocomposites synthesized via the in-situ ring-opening polymerization. Appl. Surf. Sci. 2018, 440, 1143-1149. [CrossRef] 
10. Feng, X.; Wang, B.; Wang, X.; Wen, P.; Cai, W.; Hu, Y.; Liew, K.M. Molybdenum disulfide nanosheets as barrier enhancing nanofillers in thermal decomposition of polypropylene composites. Chem. Eng. J. 2016, 295, 278-287. [CrossRef]

11. Zhou, K.; Gao, R.; Gui, Z.; Hu, Y. The effective reinforcements of functionalized MoS2 nanosheets in polymer hybrid composites by sol-gel technique. Compos. Part A 2017, 94, 1-9. [CrossRef]

12. Naffakh, M.; Díez-Pascual, A.M.; Marco, C.; Ellis, G.; Gómez-Fatou, M.A. Opportunities and challenges in the use of inorganic fullerene-like nanoparticles to produce advanced polymer nanocomposites. Prog. Polym. Sci. 2013, 38, 1163-1231. [CrossRef]

13. Naffakh, M.; Díez-Pascual, A.M. Thermoplastic polymer nanocomposites based on inorganic fullerene-like nanoparticles and inorganic nanotubes. Inorganics 2014, 2, 291-312. [CrossRef]

14. Lalwani, G.; Henslee, A.M.; Farshid, B.; Parmar, P.; Lin, L.; Qin, Y.-X.; Kasper, F.K.; Mikos, A.G.; Sitharaman, B. Tungsten disulfide nanotubes reinforced biodegradable polymers for bone tissue engineering. Acta Biomater. 2013, 9, 8365-8373. [CrossRef] [PubMed]

15. Naffakh, M.; Marco, C.; Ellis, G.; Cohen, S.R.; Laikhtman, A.; Rapoport, L.; Zak, A. Novel poly(3-hydroxybutyrate) nanocomposites containing $\mathrm{WS}_{2}$ inorganic nanotubes with improved thermal, mechanical and tribological properties. Mater. Chem. Phys. 2014, 147, 273-284. [CrossRef]

16. Naffakh, N.; Díez-Pascual, A.M. Nanocomposite biomaterials based on poly(ether-ether-ketone) (PEEK) and $\mathrm{WS}_{2}$ inorganic nanotubes. J. Mater. Chem. B 2014, 2, 4509-4520. [CrossRef]

17. Naffakh, M.; Marco, C.; Ellis, G. Development of novel melt-processable biopolymer nanocomposites based on poly(L-lactic acid) and $\mathrm{WS}_{2}$ inorganic nanotubes. CrystEngComm 2014, 16, 5062-5072. [CrossRef]

18. Karavelidis, V.; Karavas, E.; Giliopoulos, D.; Papadimitriou, S.; Bikiaris, D. Evaluating the effects of crystallinity in new biocompatible polyester nanocarriers on drug release behavior. Int. J. Nanomed. 2011, 6, 3021-3032.

19. Jabbarzadeh, A. The origins of enhanced and retarded crystallization in nanocomposite polymers. Nanomaterials 2019, 9, 1472. [CrossRef]

20. Kumar, S.K.; Ganesan, V.; Riggleman, R.A. Perspective: Outstanding theoretical questions in polymer-nanoparticle hybrids. J. Chem. Phys. 2017, 147, 020901. [CrossRef]

21. Jabbarzadeh, A.; Halfina, B. Unravelling the effects of size, volume fraction and shape of nanoparticle additives on crystallization of nanocomposite polymers. Nanoscale Adv. 2019, 1, 4704-4721. [CrossRef]

22. Luo, Y.; Lin, Z.; Guo, G. Biodegradation assessment of poly (lactic acid) filled with functionalized titania nanoparticles $\left(\mathrm{PLA} / \mathrm{TiO}_{2}\right)$ under compost conditions. Nanoscale Res. Lett. 2019, 14, 56. [CrossRef] [PubMed]

23. Fukushima, K.; Abbate, C.; Tabuani, D.; Gennari, M.; Camino, G. Biodegradation of poly(lactic acid) and its nanocomposites. Polym. Degrad. Stab. 2009, 94, 1646-1655. [CrossRef]

24. Someya, Y.; Kondo, N.; Shibata, M. Biodegradation of poly(butylene adipate-co-butylene terephthalate)/layered-silicate nanocomposites. J. Appl. Polym. Sci. 2007, 106, 730-736. [CrossRef]

25. Fukushima, K.; Abbate, C.; Tabuani, D.; Gennari, M.; Rizzarelli, P.; Camino, G. Biodegradation trend of poly( $\varepsilon$-caprolactone) and nanocomposites. Mater. Sci. Eng. C 2010, 30, 566-574. [CrossRef]

26. Fischer, E.W.; Sterzel, H.J.; Wegner, G. Investigation of the structure of solution grown crystals of lactide copolymers by means of chemical reactions. Kolloid Z. Z. Polym. 1973, 251, 980-990. [CrossRef]

27. Jarerat, A.; Pranamuda, H.; Tokiwa, Y. Poly(L-lactide)-degrading activity in various actinomycetes. Macromol. Biosci. 2002, 2, 420-428. [CrossRef]

28. Krikorian, V.; Pochan, D.J.; Krikorian, V.; Pochan, D.J. Unusual crystallization behavior of organoclay reinforced poly(l-lactic acid) nanocomposites. Macromolecules 2005, 38, 6520-6527. [CrossRef]

29. Wu, C.H.; Eder, G.; Janeschitz-Kriegl, H. Polymer crystallization dynamics, as reflected by differential scanning calorimetry. Part 2: Numerical simulations. Colloid Polym. Sci. 1993, 271, 1116-1128. [CrossRef]

30. Burzic, I.; Pretschuh, C.; Kaineder, D.; Eder, G.; Smilek, J.; Masilko, J.; Kateryna, W. Impact modification of PLA using biobased biodegradable PHA biopolymers. Eur. Polym. J. 2019, 114, 32-38. [CrossRef]

31. Ning, N.; Fu, S.; Zhang, W.; Chen, F.; Wang, K.; Deng, H.; Zhang, Q.; Fu, Q. Realizing the enhancement of interfacial interaction in semicrystalline polymer/filler composites via interfacial crystallization. Prog. Polym. Sci. 2012, 37, 1425-1455. [CrossRef]

32. Jing, M.; Jiang, H.; Guo, Y.; Wu, Z.; Fu, Q. Transcrystallization of poly(l-lactic acid) on the surface of reduced graphene oxide fibers. RSC Adv. 2016, 6, 100090-100097. [CrossRef] 
33. Pluta, M.; Galeski, A.; Alexandre, M.; Paul, M.A.; Dubois, P. Polylactide/montmorillonite nanocomposites and microcomposites prepared by melt blending: Structure and some physical properties. J. Appl. Polym. Sci. 2002, 86, 1497-1506. [CrossRef]

34. Safandowsk, M.; Rozanski, A.; Galeski, A. Plasticization of polylactide after solidification: An effectiveness and utilization for correct interpretation of thermal properties. Polymers 2020, 12, 561. [CrossRef]

35. Liu, T.; Mo, Z.; Wang, S.; Zhang, H. Nonisothermal melt and cold crystallization kinetics of poly(aryl ether ether ketone ketone). Polym. Eng. Sci. 1997, 37, 568-575. [CrossRef]

36. Avrami, M. Kinetics of phase changes 1. General theory. J. Chem. Phys. 1939, 7, 1103-1112. [CrossRef]

37. Avrami, M. Kinetics of phase change. II. Transformation-time relations for random distribution of nuclei. J. Chem. Phys. 1940, 8, 212-224. [CrossRef]

38. Avrami, M. Kinetics of phase change. III. Granulation, phase change, and microstructure. J. Chem. Phys. 1941, 9, 177-184. [CrossRef]

39. Ozawa, T. Kinetics of non-isothermal crystallization. Polymer 1971, 128, 150-158. [CrossRef]

40. Naffakh, M.; Marco, C.; Gómez, M.A.; Jiménez, I. Unique nucleation activity of inorganic fullerene-like WS2 nanoparticles in polyphenylene sulfide nanocomposites: Isokinetic and isoconversional study of dynamic crystallization kinetics. J. Phys. Chem. B 2009, 113, 7107-7115. [CrossRef]

41. Kissinger, H.E. Variation of peak temperature with heating rate in differential thermal analysis. J. Res. Natl. Bur. Stand. 1956, 57, 217-221. [CrossRef]

Publisher's Note: MDPI stays neutral with regard to jurisdictional claims in published maps and institutional affiliations.

(C) 2020 by the authors. Licensee MDPI, Basel, Switzerland. This article is an open access article distributed under the terms and conditions of the Creative Commons Attribution (CC BY) license (http://creativecommons.org/licenses/by/4.0/). 